\title{
Haul road defect identification using measured truck response
}

\author{
D. Hugo ${ }^{\text {a }}$, P.S. Heyns ${ }^{\text {a,* }}$, R.J. Thompson ${ }^{\text {b }}$, A.T. Visser ${ }^{c}$ \\ ${ }^{a}$ Dynamic Systems Group, Department of Mechanical and Aeronautical Engineering, University of Pretoria, Pretoria 0002, South Africa \\ ${ }^{\mathrm{b}}$ Department of Mining Engineering, University of Pretoria, Pretoria 0002, South Africa \\ ${ }^{\mathrm{c}}$ Department of Civil Engineering, University of Pretoria, Pretoria 0002, South Africa
}

Received 9 September 2006; received in revised form 21 November 2007; accepted 23 July 2008

\begin{abstract}
Current management techniques for the maintenance of mine haul roads, such as ad hoc blading, scheduled blading and even maintenance management systems, have shortcomings in complex mining environments. This paper investigates the possibility of using the response of haul trucks to aid the management of haul road maintenance. The question arises as to whether truck response data can be used to recognize road defects at specific locations, in terms of type and size. This is important since different defect types require different road maintenance strategies. A modeling methodology based on dynamic equilibrium of the unsprung mass of a haul truck is proposed and investigated.
\end{abstract}

(C) 2008 ISTVS. Published by Elsevier Ltd. All rights reserved.

Keywords: Off-road vehicle; Mine haul truck; Road maintenance; Road management

\section{Introduction}

The high rates at which haul roads in opencast mines deteriorate necessitate intensive maintenance and make the management of this maintenance important. Too little road maintenance leads to excessive cost in the operation and maintenance of vehicles, whereas excessive road maintenance costs more but does little to reduce the cost of operation and maintenance of vehicles. Existing maintenance techniques such as ad hoc blading and scheduled blading fail to optimize this complex cost function [1].

Thompson and Visser [2] developed a maintenance management system applicable to mine haul roads. This includes visual assessment that rates road defects according to their types and degrees of severity. The sum of the defect ratings for a segment of road constitutes a defect score which is related to rolling resistance through an empirical model. Every cost factor, such as fuel consumption, tire wear and vehicle maintenance on the one hand, and road

\footnotetext{
${ }^{*}$ Corresponding author. Tel.:+2712 420 2432; fax: +2712 3625087.

E-mail address: stephan.heyns@up.ac.za (P.S. Heyns).
}

maintenance costs on the other hand, is related empirically to the road defect score. The road deterioration rate after maintenance is also modeled. By combining and optimizing these empirical models, it becomes possible to determine the optimal maintenance interval.

Maintenance management systems such as these rely heavily on accurate modeling input for their success. Thompson et al. [1] demonstrate that this technique can be applied relatively easily to situations where segment characteristics are quasi-static and rapid changes can be predicted. Examples of this include hauling from another ramp, a change in tonnage hauled between ramps, etc. For complex road networks where material is sourced and hauled from a large and highly variable number of loading points, repeated remodeling is required with no guarantee that the variables assumed (e.g. traffic volume) will be realized.

Recognizing the shortcomings of the current management techniques for mine haul road maintenance, this paper investigates the possibility of using measured haul truck response as an aid in the management of haul road maintenance. 


\section{Haul truck response as a tool for road maintenance management}

Paved road users and the agencies maintaining the roads are normally independent. It is therefore not surprising that paved road maintenance agencies use dedicated vehicles for the sole purpose of profiling. Since the concept of total cost applies to the mining industry [1], it however becomes appropriate to investigate the possible use of operational mine vehicles for road maintenance management purposes as well. This might be even more viable because it is becoming increasingly common to install communication and positioning equipment on haul trucks, to communicate performance data and truck locations to a maintenance dispatch office on a continuous basis.

The use of operational trucks also features other advantages compared to specialized profilers. Firstly, since these vehicles operate in their design environment there is no cause for concern about using fragile profilers in harsh environments. Secondly, these vehicles can capture road condition data while operating. This is a major advantage in view of the need for frequent assessment.

There are also some disadvantages when using operational vehicles. Specialized profilers can be designed and selected to have the most desirable properties for the purpose of road profiling. Operational vehicles are optimized for other objectives. Moreover, the primary function of operational vehicles (hauling material) probably requires less stringent control over dynamic response properties than would be the case for some profiling purposes. Consequently, there might be difficulty in establishing adequate levels of control over vehicle properties, such as suspension strut pressure, tire pressure and load.

Compared to existing haul road maintenance management techniques, truck response measurements have the potential for thorough, yet less labor-intensive assessment. Every stretch of road the truck drives over may be assessed. Frequent visual inspection of long sections of road would no longer be necessary since the truck response can be measured and processed continuously and automatically. The quality of assessment will primarily dependent on the effectiveness of the analysis techniques used. The inherent objectivity of truck response measurements is a second major advantage. Most of the existing techniques entail some form of visual inspection. Even maintenance management systems are derived from subjective evaluations. Finally, truck response measurements, as opposed to maintenance management systems, also have the potential not only to identify localized defects, but also to record the exact locations of these road defects by means of the truck global positioning system (GPS).

The ability to identify localized defects in haul roads is desirable to minimize vehicle operating and maintenance costs. In addition the ability to classify defects in terms of type and size would be advantageous, since the types and sizes of defects determine the appropriate maintenance strategies. This paper adopts a mathematical vehicle mod- eling approach and utilizes measured haul truck response in pursuit of the challenge to reconstruct the geometries of large defects as an input to guide decisions about road maintenance.

The second approach by which haul truck response might be utilized is to produce objective ratings of the international roughness index (IRI) by measuring the motion of the suspension strut of the haul truck. Many maintenance management systems currently use subjective IRI ratings in their calculations, which is obviously inferior to measured values. This approach will however not be dealt with in this discussion.

\section{Road input reconstruction methodology}

The combination of inputs from different sources produces complex vehicle-weighted responses. Use of the vehicle response to identify road input therefore requires differentiation between the response caused by road input and the response induced by other sources. This seems to be a reasonable objective since responses from road inputs and other sources mainly appear in different frequency ranges. However, the measurement locations can be chosen to minimize the influence from unwanted excitation sources.

Since a typical haul truck features hydro-pneumatic suspension struts that exhibit non-linear spring and damping characteristics, measuring anywhere on the sprung mass would require inversion of a non-linear system in order to accurately calculate road input from measured response.

To avoid inversion of the non-linear system, the road input could in principle be reconstructed by iteratively changing the road elevation provided to the simulation model, until the simulated response matches the measured response. Still however, characterization of the complete vehicle would be necessary.

For these reasons, a simple model is required, which will also favor real time on-board calculation of the road input and enable localization of road defects, using the on-board GPS. In addition the model has to be as general as possible to simplify portability between different truck models.

To satisfy these demands, a very simple model of a typical haul truck front wheel and suspension was formulated. Fig. 1 depicts the rigid free-body diagram of an independent front unsprung mass. If the acceleration of the unsprung mass $\ddot{z}_{u}$ and the force acting between the sprung and unsprung masses $F_{\text {suspension }}$ are measured, the road force $F_{\text {tire }}$ can be calculated from the simple algebraic equation

$F_{\text {tire }}=m_{u} \ddot{z}_{u}+F_{\text {suspension }}$

The model suggested in Fig. 1 treats the suspension strut force as a known variable. Consequently, this force is not modeled as a non-linear function of relative suspension strut displacement and velocity, nor is the inversion of a non-linear system required. Most importantly, the characterization of the complete vehicle becomes unnecessary. 


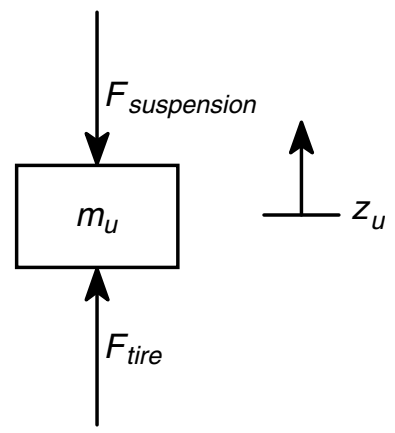

Fig. 1. Dynamic equilibrium of an independent front unsprung mass $m_{\mathrm{u}}$ of the haul truck.

Assuming the suspension force to be a measurable variable (see paragraph 4), one approach to calculate the road input would be to enforce dynamic equilibrium on the unsprung mass and iteratively change the road input until the simulated unsprung mass acceleration response $\ddot{z}_{u}$ matches the measured response. This approach can accommodate complex tire models since no inversion is required.

The approach adopted for this study however, calculates the tire force $F_{\text {tire }}$ directly through Eq. (1). This force is then used together with the unsprung mass displacement and an inverse tire model to calculate the corresponding road input.

The advantage of this approach is that neither structural vibrations nor vibrations from other on-board sources significantly influence the measured signals in the frequency range below $10 \mathrm{~Hz}$. This will be proven through detailed analysis of the measured data on the truck under consideration.

Tire/wheel non-uniformities create forces that cannot be distinguished from the tire force $F_{\text {tire }}$. However, Cebon [3] indicates that forces caused by tire non-uniformities are usually only significant on relatively smooth roads, where they can be comparable in magnitude to forces caused by road excitation. Since the present work is aimed at identifying large defects on gravel roads, these effects may be considered negligible. All other significant forces acting on the unsprung mass, such as those caused by the pitch and roll motion of the sprung mass, are transferred through the suspension. These can therefore be measured separately from the tire force.

This method corresponds in principle to the straingauged axle method described by Cebon [3]. The latter uses strain gauges to measure either the bending moments or shear forces of an axle between the spring mountings and wheels, and accelerometers to measure the acceleration of the axle. Dynamic equilibrium is then enforced on the components outboard of the strain gauges to calculate the tire force. The main difference between the method proposed here and that described by Cebon is the way in which the forces between the unsprung and sprung masses are measured.

\section{Vehicle characterization and dynamic response measurements}

Practical measurements were done on a Komatsu 730E Haulpack (Fig. 2). This vehicle has a GVW (Gross Vehicle Weight) of 324 tons, a load capacity of 186 tons, a rated engine power of $1492 \mathrm{~kW}$ and an inflated tire diameter of about $3.5 \mathrm{~m}$. The vehicle is powered at the rear wheels by two electrical motors located in a hollow cylindrical housing. Two hydro-pneumatic struts suspend the sprung mass of the vehicle at the rear. Part of the rear suspension is a trailing arm that pivots on the sprung mass. At the front of the vehicle, the sprung mass is suspended by two independent hydro-pneumatic suspension struts. The left and right front unsprung masses can therefore move independently. Although various models of haul trucks differ significantly from each other, this truck can be considered quite typical and the techniques presented here could quite easily be generalized to haul trucks of this nature.

The haul truck in Fig. 2 was characterized in terms of its dynamic response properties. This comprised the characterization of the vertical stiffness of the tires, as well as the damping and stiffness of the front suspension struts. Furthermore, the dynamic response of an operational vehicle was measured on a test section that contained specially constructed artificial defects.

\subsection{Vehicle characterization}

The truck tires had to be characterized in situ on an open-air weighbridge because of their size. The truck was loaded with different quantities of coal, while recording the weight on each wheel. At the same time the vertical displacement between the ground and a point on the rim of each wheel was measured, using the unladen tire radius as zero. To obtain one more data point, tire deflection was also measured under the tire's own weight. This procedure was repeated for two different tire inflation pressures.



Fig. 2. The Komatsu 730E Haulpack used in the study. 
It was however found that slight pressure variations and the use of tires from different manufacturers, did not cause significant variation in the observed stiffness characteristics. The data was therefore deemed adequate to calculate a vertical tire stiffness characteristic to serve the current modeling requirements. A reasonably linear relationship was evident over the range of measurement and an effective value for tire stiffness was calculated.

The hydro-pneumatic suspension struts contain nitrogen gas and oil. The compression of the nitrogen gas causes a spring force, whereas the resistance of the oil flow through the damping ports causes a damping force. Such a front suspension strut was characterized while supported vertically as shown in Fig. 3. A $630 \mathrm{kN}$ actuator was used to excite it over a range of frequencies. During excitation the total force exerted by the strut was measured by the actuator load cell, the strut displacement was measured by the linear variable displacement transducer of the actuator, and the nitrogen gas pressure inside the strut was measured by a standard pressure transducer.

The measured gas pressure and the effective area inside the strut on which the gas acted, were used to calculate the spring force exerted by the strut. Subsequently, the ideal gas law was used to characterize the stiffness of the strut in terms of spring force versus displacement. This characteristic was also calculated from the dynamic response data (nitrogen gas pressure and strut displacement) measured on an operational vehicle. It was found that adiabatic compression resembled the motion of the

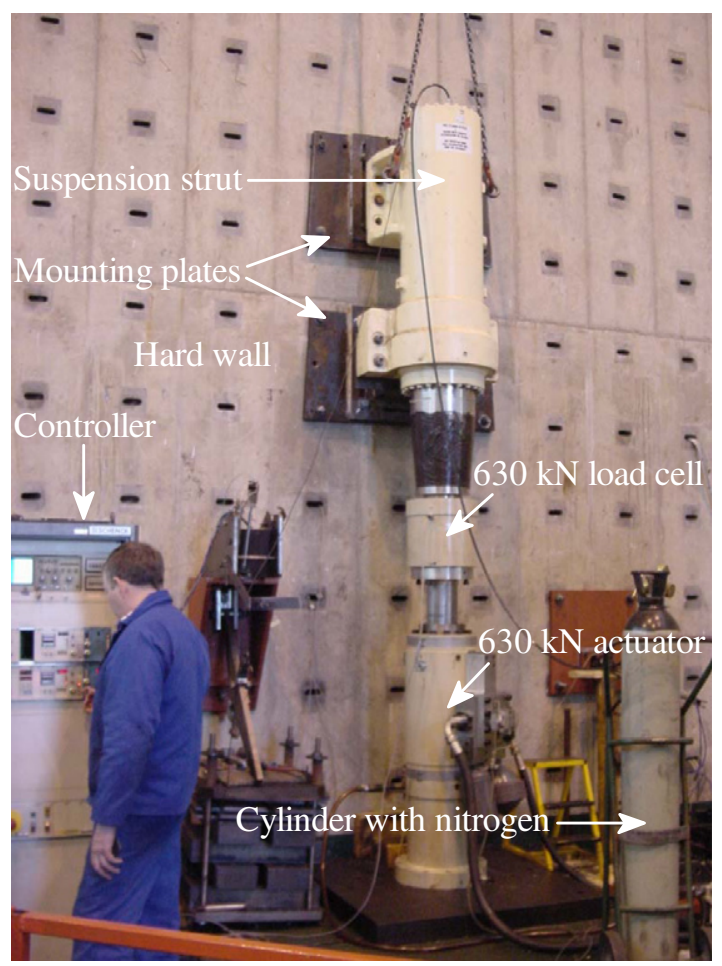

Fig. 3. Test setup for the characterization of the Komatsu 730E Haulpack suspension strut.

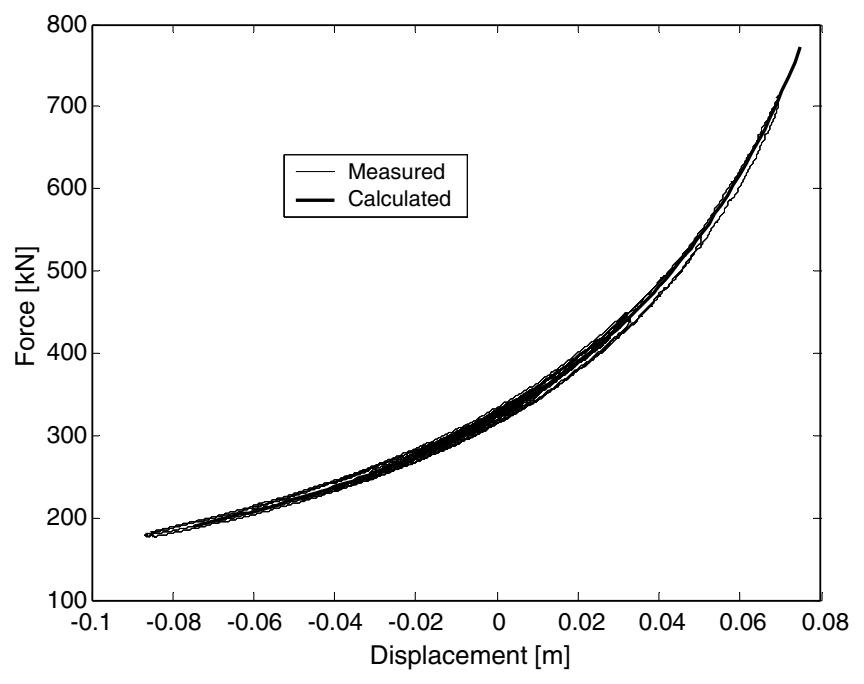

Fig. 4. Measured and calculated characteristics of suspension strut stiffness.

struts during operation of the vehicle quite well. Fig. 4 depicts the calculated adiabatic characteristic superimposed on the measured data. Els [4] presents a method for modeling the hysteretic nature of the measured data which is due to heat transfer effects. For the purposes of this work however, the characteristic presented in Fig. 4 was considered sufficient.

Since the stiffness characteristic could be calculated from the operational vehicle response data, the primary purpose of the laboratory measurements was to characterize the strut in terms of its damping properties, which could not be done from the quantities measurable on the operational vehicle. Exciting the strut with sawtooth displacement signals over a range of frequencies created different constant velocity compression and extension cycles. During excitation the damping force exerted by the strut was calculated as the difference between the total force and the spring force. The resulting force therefore includes a friction component. The relative strut velocity during compression and extension at two instants during excitation at each frequency, were then plotted against the corresponding damping forces to obtain the damping characteristic of the strut over a range of velocities.

The rate of hydraulic oil flow to the actuator limited the absolute strut velocity in the laboratory to $0.06 \mathrm{~m} / \mathrm{s}$. Since the relative velocity of a strut on an operational vehicle frequently exceeds this value when the vehicle encounters defects on the road, it was necessary to extrapolate the measured characteristic. The fluid damping in this strut is caused by ports with simple geometries. As a result, it was possible to describe the pressure drop over these ports in terms of the fluid velocity through the ports. By expanding the theoretical relationships, it was finally possible to describe the damping force exerted by the strut in terms of relative strut velocity as 
$F_{\text {damping }}=C_{\text {damping }} V_{\text {suspension }}^{2}$

where $F_{\text {damping }}$ is the damping force, $V_{\text {suspension }}$ is the suspension strut velocity and $C_{\text {damping }}$ is a constant that consists of known parameters.

Fig. 5 displays the measured and calculated damping characteristics. Eq. (2) was used to produce the calculated characteristic with different values for $C_{\text {damping }}$ in compression and extension. Higher damping in extension compared to compression is evident, which is consistent with the strut design in which some of the ports are closed during extension. Even though the relationship between pressure drop and fluid velocity was significantly simplified by assuming incompressible steady-state one-dimensional frictionless flow, it is evident from this figure that Eq. (2) resembles the measured data well up to $0.06 \mathrm{~m} / \mathrm{s}$ The rest of the characteristic can only be verified through a detailed analysis or measurement over the complete operating range of the strut. Results were found to be fairly insensitive to minor changes in the damping properties. This characteristic was therefore deemed adequate for the current purposes.

The dynamic friction force that is present when the strut is in relative motion is represented by the offsets from the zero position of the compression and extension curves in Fig. 5. This force might change slightly during operation, but only by amounts which are negligible in the current application, and is therefore modeled as part of the damping force by simply adding a constant force value to the equations that describe fluid damping during compression and extension.

The magnitude of the static friction force present when the relative strut motion is zero cannot be determined in terms of relative strut motion, implying that very small road defects or low roughness levels will not be identifiable when using this approach to calculating the suspension strut force.

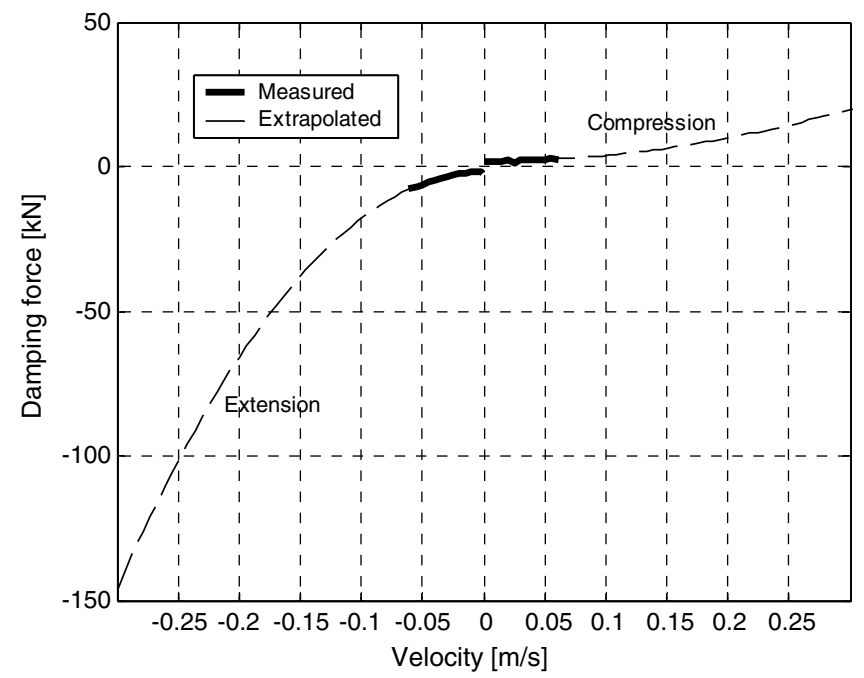

Fig. 5. Measured and extrapolated characteristics of suspension strut damping.

\subsection{Dynamic response measurements}

To investigate the methodology introduced earlier, measurements on the operational vehicle were focused on the front of the vehicle. The measurands included the vertical acceleration of the independent front unsprung masses, the vertical acceleration of the sprung mass at the front (measured on top of the suspension struts), the front suspension strut pressures, the relative displacement of the front struts, and the pitch, roll and yaw velocities of the sprung mass.

Measuring these quantities, the unladen truck was driven over a test section with artificially constructed defects, a few times at different speeds. The defects were comprised of ditches and humps ranging in width from 0.6 to $7.8 \mathrm{~m}$ and in height (or depth) from 0.1 to $0.35 \mathrm{~m}$. The position of the vehicle on the road was recorded by a GPS, and the locations of the defects were established in the same coordinate system prior to taking the response measurements. This enabled the response measurements to be synchronized with the road input as the truck traversed the road section.

With these data sets available, mathematical modeling and simulation became possible. The modeling formed a basis for understanding the problem, as well as enabling the vehicle characteristics to be verified and the accuracy of suspension strut force measurement to be assessed.

\section{Vehicle modeling and the rigid-body assumption}

Two rigid-body vehicle models were developed for investigative studies: a single degree-of-freedom (1DOF) quarter-vehicle model and a seven degree-of-freedom (7DOF) vehicle model. The tire and suspension properties discussed previously were used in these models.

The 1DOF model represented a front corner of the haul truck using the front tire and suspension characteristics. The tire was simulated by means of a circular rigid treadband tire model (Fig. 6) [5]. This model consists of a linear spring and a rigid ring representing the treadband, which models the trajectory of the tire over the road more accurately than the basic point-follower model. This tire model

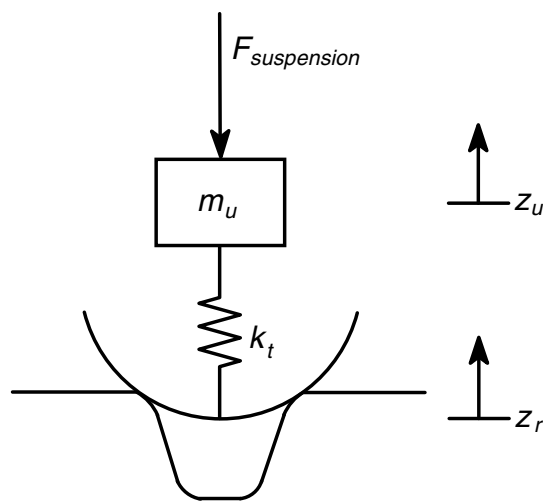

Fig. 6. Graphical illustration of the circular rigid treadband tire model. 
(a) Sprung mass acceleration

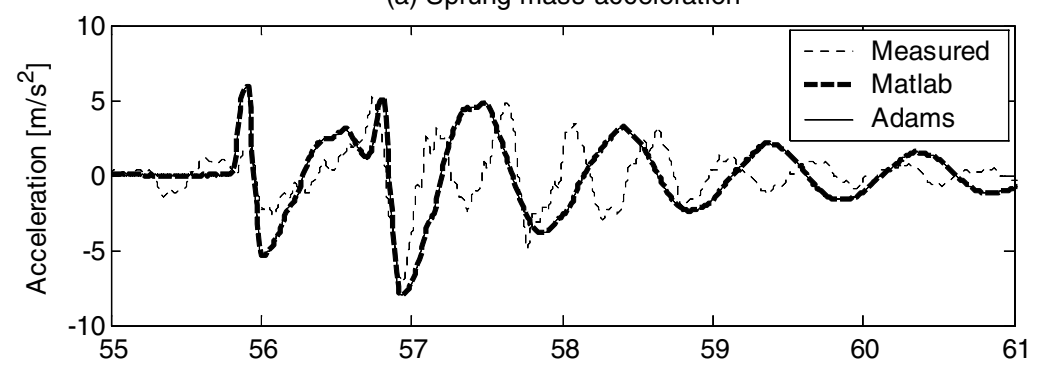

(b) Unsprung mass acceleration

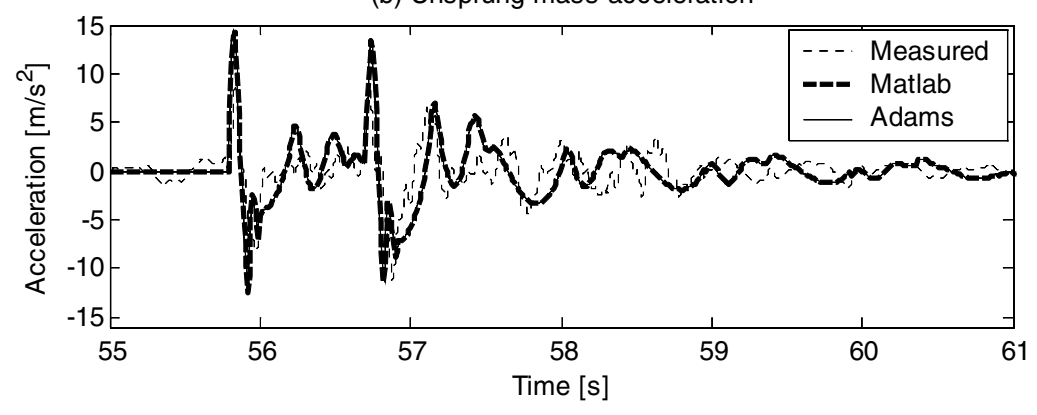

Fig. 7. Single-degree-of-freedom (1DOF) vehicle modeling results.

was implemented by filtering the road profile by repeated lowering of the tire from a vertically elevated position, until any point on the treadband touches the road. The corresponding elevations of the center of the treadband was then determined over the length of the road. This profile then established the input into the 1DOF simulation model which represented the tire stiffness as a simple spring.

Fig. 7 superimposes the simulated sprung and unsprung mass accelerations (using a Matlab and an Adams model) on measured acceleration responses while traversing two half-sine humps separated by a distance of $6 \mathrm{~m}$ at approximately $20 \mathrm{~km} / \mathrm{h}$. It is evident that the initial accelerations when encountering the first hump were accurately modeled, whereas the subsequent transient motions were not.

The 7DOF vehicle model allowed vertical, pitch and roll motion of the sprung mass, vertical and roll motion of the rear unsprung mass (which can be regarded as a solid axle for the current modeling purposes) and vertical motion of the two independent front unsprung masses. Since the rear suspension strut damping and stiffness characteristics were

(a) Sprung mass acceleration

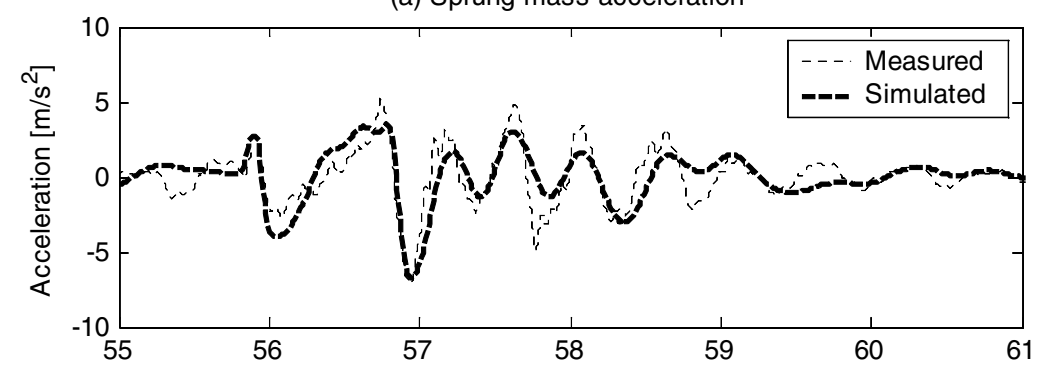

(b) Unsprung mass acceleration

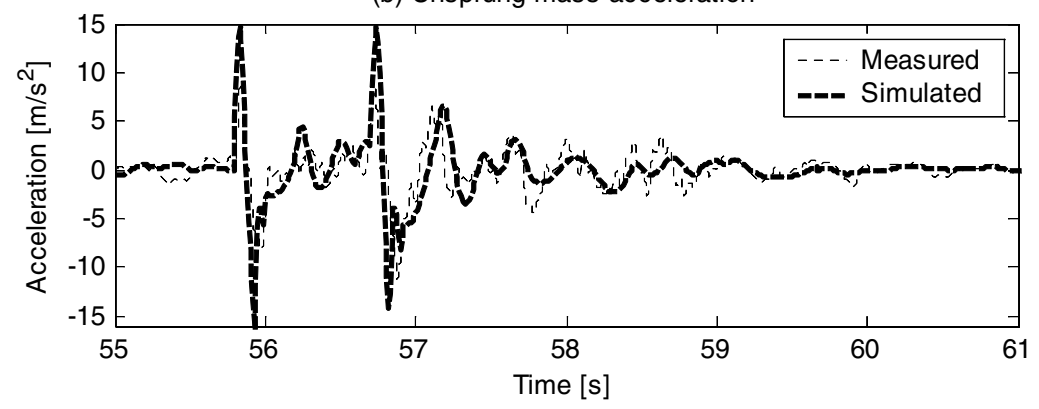

Fig. 8. Seven-degrees-of-freedom (7DOF) vehicle modeling results. 
not determined, the equations of motion describing this model were manipulated to eliminate these forces, treating the measured rotational accelerations as known variables. In Fig. 8 simulated results (Matlab model) and measured accelerations are shown. The 7 DOF model clearly captures the dynamic response much better than the 1DOF model.

The undamped bounce natural frequency of the $1 \mathrm{DOF}$ model can be approximated by linearizing its properties around the static equilibrium position as

$f_{\mathrm{ns}}=\frac{1}{2 \pi} \sqrt{\frac{\left(\frac{k_{\mathrm{s}} k_{\mathrm{t}}}{k_{\mathrm{s}}+k_{\mathrm{t}}}\right)}{M_{\mathrm{s}}}}=1 \mathrm{~Hz}$

where the suspension spring stiffness $k_{\mathrm{s}}=2.6 \times 10^{6} \mathrm{~N} / \mathrm{m}$, the tire stiffness $k_{\mathrm{t}}=2.7 \times 10^{6} \mathrm{~N} / \mathrm{m}$ and the sprung mass $M_{\mathrm{s}}=32895 \mathrm{~kg}$. Fig. 9a shows the power spectral density (PSD) of the vertical acceleration of the sprung mass, measured on top of the front suspension strut. The peak at $1 \mathrm{~Hz}$ corresponds to the bounce natural frequency calculated in Eq. (3).

Fig. 9a shows a second peak just below $2 \mathrm{~Hz}$ and the PSD of the measured pitch acceleration of the sprung mass (Fig. 9b) shows peaks at the same frequencies of respectively, 1 and $2 \mathrm{~Hz}$ Simple analysis following Gillespie [6] shows that the amplitude ratios of the bounce and pitch motion at these frequencies have opposite signs (Fig. 10), and therefore represent the bounce and pitch modes of the vehicle, respectively.

Vehicle pitch is therefore responsible for the dominant motion at $2 \mathrm{~Hz}$ (Fig. 8), just after the vehicle has passed a defect. Furthermore, it is clear that even though the static friction component is neglected, the suspension strut forces are calculated quite accurately.
Since the bounce and pitch modes of the vehicle are the only significant peaks in the vertical acceleration PSD below $10 \mathrm{~Hz}$ (Fig. 9.a), the rigid-body assumption for the sprung mass acceleration measurements on top of the suspension strut seems clearly justified.

The pitch acceleration PSD (Fig. 9b) shows significant response at higher frequencies. This signal was obtained by differentiating the pitch velocity measured by a gyroscope located on the frame of the truck. Although there is no certainty about the cause of these large responses, it is evident that non-rigid body motion dominates in the higher frequency region. Consequently, a cut-off frequency of $3 \mathrm{~Hz}$ was specified for the measured pitch acceleration that was used in the 7DOF model that produced the results in Fig. 8. A cut-off frequency of $1.5 \mathrm{~Hz}$ was used for the measured roll acceleration.

The PSD of vertical acceleration measured on the spindle of the front unsprung mass also showed response at higher frequencies than the sprung mass. This was to be expected, since wheel hop generally appears at a frequency higher than the bounce natural frequency. The wheel hop frequency of the independent front unsprung mass of this truck can be approximated as

$f_{\mathrm{nu}}=\frac{1}{2 \pi} \sqrt{\frac{k_{\mathrm{s}}+k_{\mathrm{t}}}{m_{\mathrm{u}}}}=3.96 \mathrm{~Hz}$

where the unsprung mass $m_{\mathrm{u}}=8578.32 \mathrm{~kg}$. It is furthermore unlikely that the spindle of the truck, which is a stiff component, would have flexural modes below $10 \mathrm{~Hz}$. Nor could any such modes be identified from the measured PSD. It is therefore concluded that the rigid-body assumption also applies to the vertical unsprung mass acceleration below $10 \mathrm{~Hz}$.

(a) PSD of sprung mass vertical acceleration

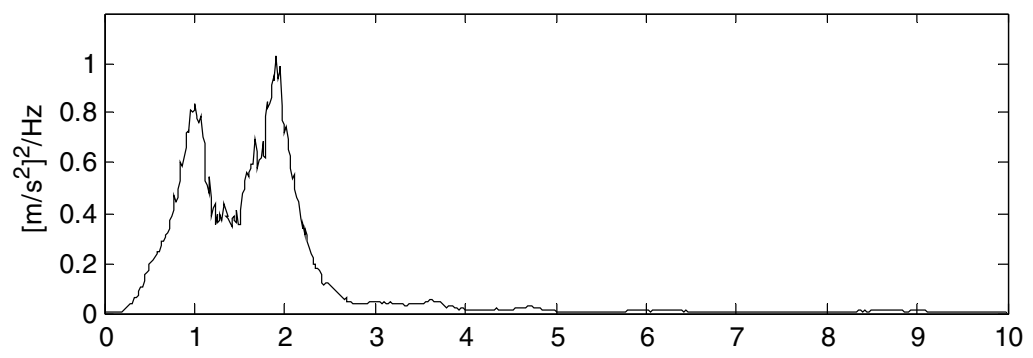

(b) PSD of sprung mass pitch acceleration

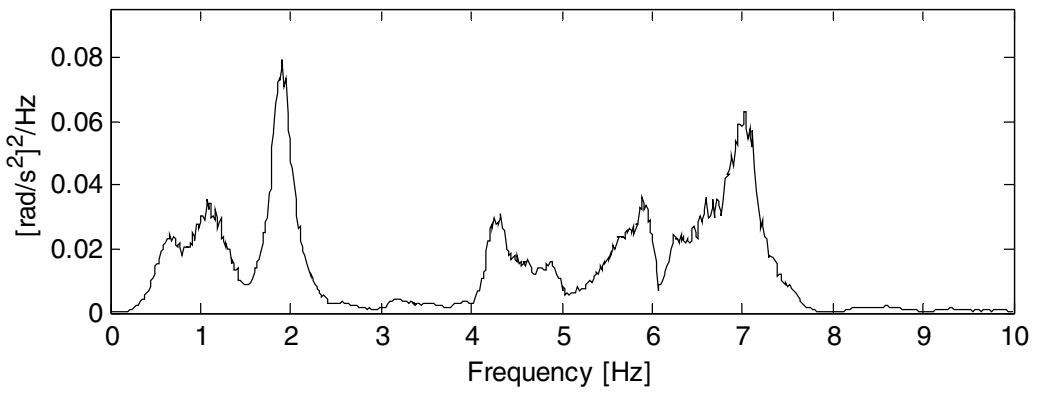

Fig. 9. Measured PSDs of sprung mass vertical and pitch acceleration. 
D. Hugo et al. I Journal of Terramechanics $x x x$ (2008) $x x x-x x x$

(a) Vertical and pitch accelerations below $1.5 \mathrm{~Hz}$

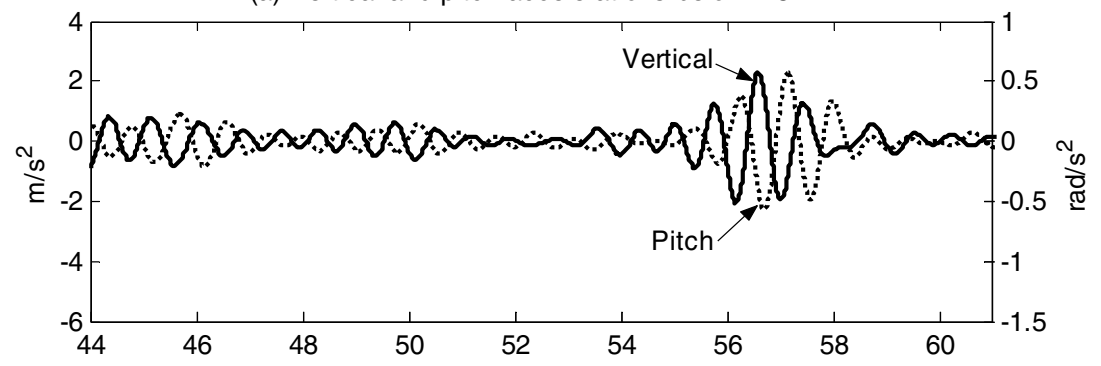

(b) Vertical and pitch accelerations between 1.5 and $3 \mathrm{~Hz}$

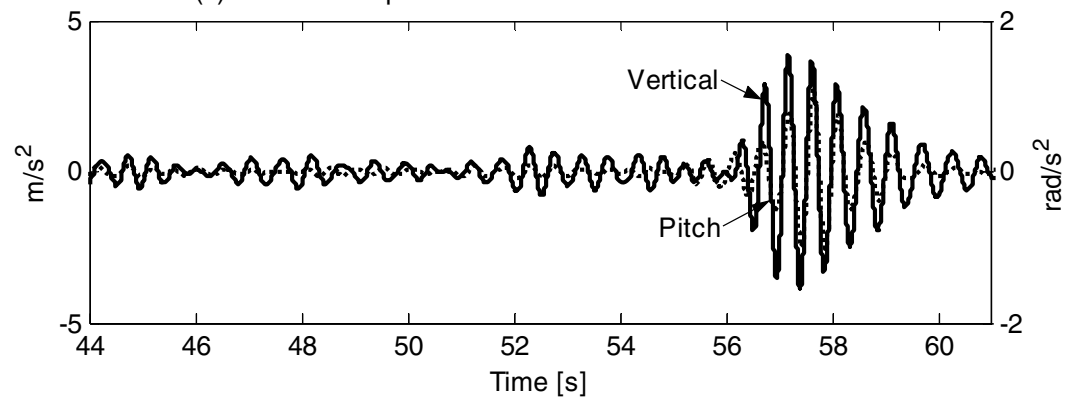

Fig. 10. Comparison of sprung mass vertical and pitch accelerations in different frequency ranges.

(a) Defect 1

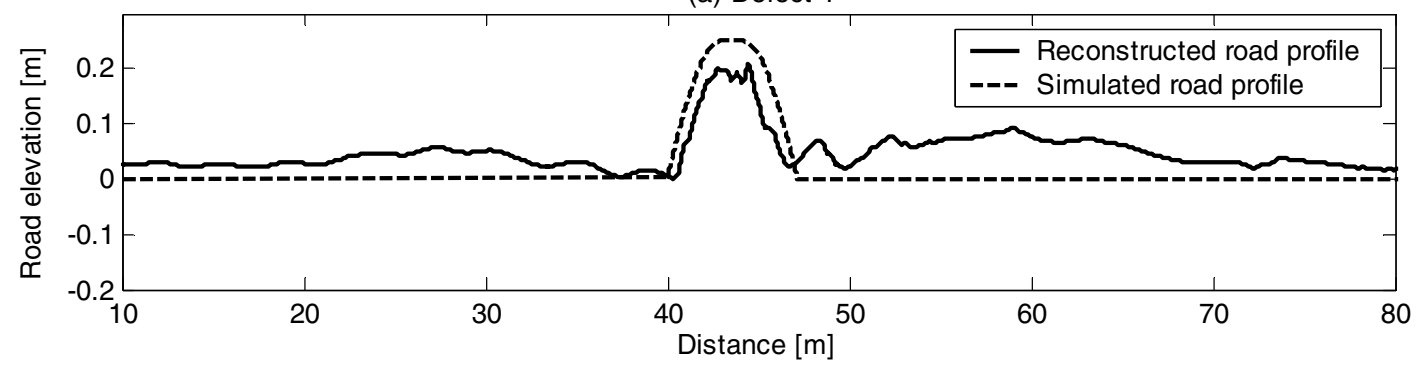

(b) Defect 2

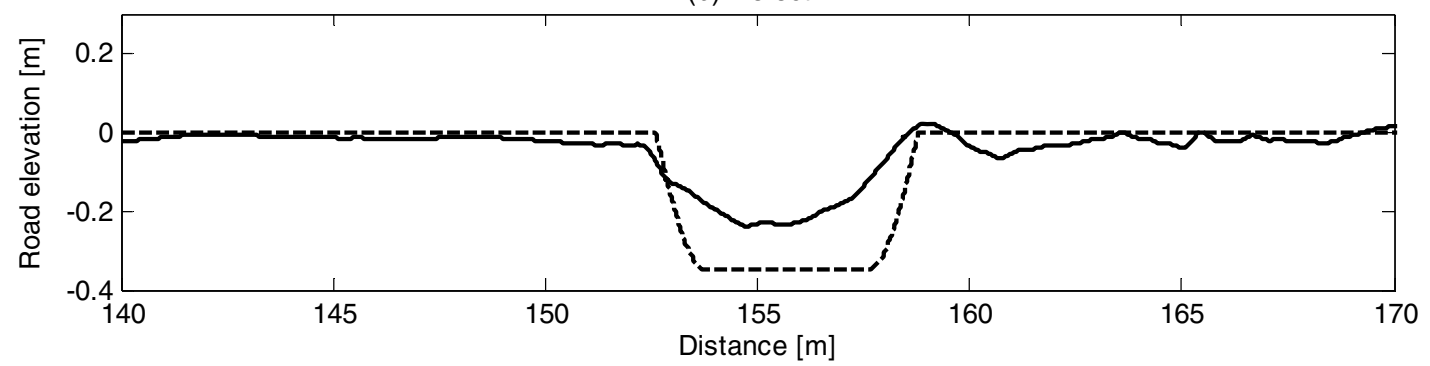

(c) Defect 3

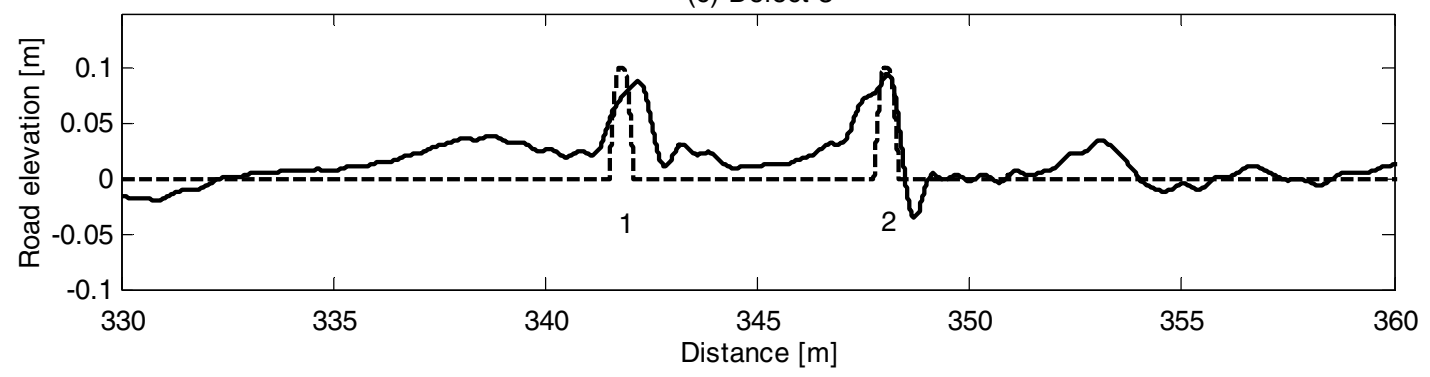

Fig. 11. Comparison of the measured and reconstructed geometries for three road defects. 


\section{Road defect reconstruction}

Working under the rigid-body assumption, defects were reconstructed. The tire force $F_{\text {tire }}$ is calculated in Eq. (1). If the tire is treated as a simple linear spring, the following equation applies

$z_{\mathrm{r}}=z_{\mathrm{u}}+\frac{F_{\text {tire }}}{k_{\mathrm{t}}}$

where $z_{\mathrm{r}}$ is the road elevation and $z_{\mathrm{u}}$ is the unsprung mass displacement.

The unsprung mass displacement is the only unknown remaining in Eq. (5). It is difficult to measure absolute displacement. The obvious alternative is to integrate the unsprung mass acceleration twice, which is bound to produce spurious low-frequency drift.

A double integration on the measured unsprung mass acceleration in the frequency domain without discarding low-frequency response resulted in a profile with severe drift. This drift was removed by fitting third-order polynomials to sections of the profile to resemble the drift without substantially changing the geometry of the defect. The curve described by this polynomial was then subtracted from the profile. The known speed of the vehicle was used to transform the resulting profile from the time domain to the spatial domain.

Figs. 11 and 12, respectively, depict three defects that were reconstructed in this way. The vehicle speed in this run was just over $20 \mathrm{~km} / \mathrm{h}$ on average and the truck was unladen.

Alternatively, removing the drift by low-pass filtering the unsprung mass displacement before reconstruction seemed to discard important low-frequency content that contributed to the shape of the defects. This made the fitting of the third-order polynomial the most attractive option.

Table 1 lists the measured, treadband-filtered and reconstructed widths and heights of the defects shown in Figs 11 and $12 . W_{\mathrm{m}}$ is the measured width, $W_{\mathrm{f}}$ is the treadband-filtered width and $W_{\mathrm{r}}$ is the reconstructed width, whereas $H_{\mathrm{m}}$ is the measured height (or depth) and $H_{\mathrm{r}}$ is the reconstructed height (or depth). The measured and treadbandfiltered defect heights are the same.

It is clear that defect reconstruction results are reasonable. Haul road defects can be identified in terms of location, type and size. However, there is still some doubt about the consistency of reconstruction. Further work that should include measurement and analysis of response over various defects at different speeds is therefore required.

(a) Defect 4

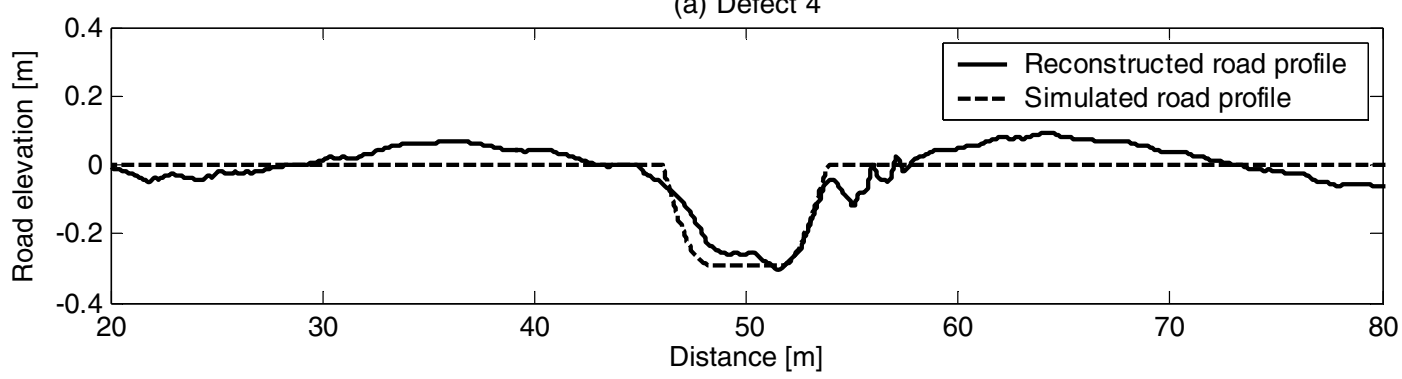

(b) Defect 5



(c) Defect 6

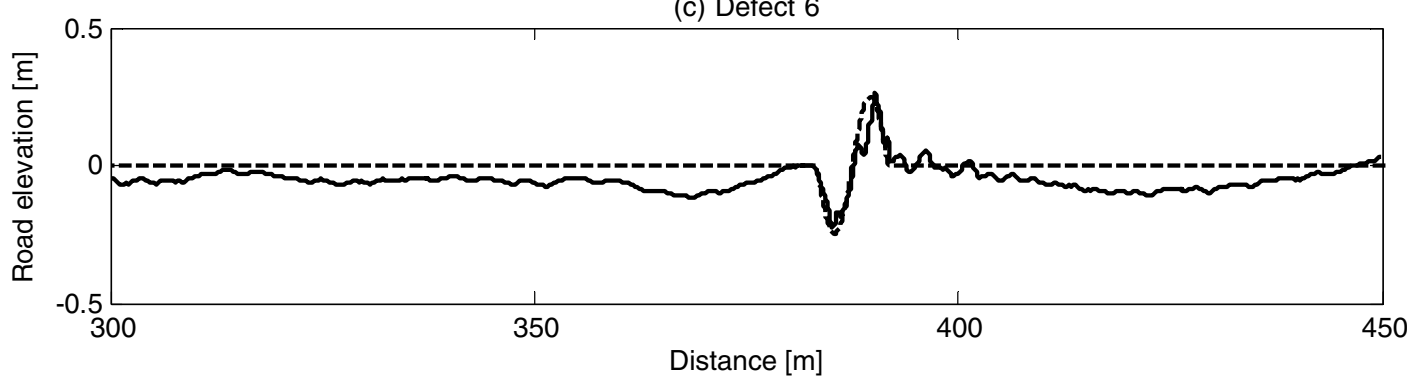

Fig. 12. Comparison of the measured and reconstructed geometries for three more defects. 
Table 1

Measured, treadband-filtered and reconstructed defect widths and heights

\begin{tabular}{|c|c|c|c|c|c|c|}
\hline & \multirow[t]{2}{*}{ Defect type } & \multicolumn{3}{|l|}{ Width } & \multicolumn{2}{|l|}{ Height } \\
\hline & & $W_{\mathrm{m}}(\mathrm{m})$ & $W_{\mathrm{f}}(\mathrm{m})$ & $W_{\mathrm{r}}(\mathrm{m})$ & $H_{\mathrm{m}}(\mathrm{m})$ & $H_{\mathrm{r}}(\mathrm{m})$ \\
\hline Defect 1 (Fig. 11a) & Hump & 7.2 & 7.43 & 6.32 & 0.25 & 0.190 \\
\hline Defect 2 (Fig. 11b) & Ditch (oblique) & 6.2 & 6.2 & 6.52 & 0.35 & 0.224 \\
\hline Defects 3-1 (Fig. 11c) & Hump & 0.6 & 1.36 & 1.60 & 0.1 & 0.070 \\
\hline Defects 3-2 (Fig. 11c) & Hump & 0.6 & 1.36 & 1.52 & 0.1 & 0.082 \\
\hline Defect 4 (Fig. 12a) & Ditch & 7.8 & 7.8 & 9.07 & 0.29 & 0.267 \\
\hline Defect 5 (Fig. 12b) & Hump & 3.2 & 3.46 & 3.56 & 0.15 & 0.151 \\
\hline Defect 6 (Fig. 12c) & Ditch \& hump & 8.4 & 8.57 & 9.15 & 0.5 & 0.489 \\
\hline
\end{tabular}

The results for the two defects in Fig. 11c gives some indication of the effect of tire enveloping on small defect reconstruction. $W_{\mathrm{r}}$ is much larger than $W_{\mathrm{m}}$.

\section{Conclusions}

The road defect reconstruction results show potential for the use of haul truck response as a management tool for haul road maintenance. The simplicity of this method is advantageous for real-time implementation and the fact that it requires basically no alteration to the vehicle is also desirable.

The method should be validated and refined through further work. This should include the refinement of algorithms, as well as the measurement and analysis of truck response over many more defects at various speeds. Lastly, incorporating more sophisticating tire models should enhance defect reconstruction accuracy.

\section{Acknowledgements}

This research was made possible through the support of our industry partners, Kumba Resources and De
Beers Venetia Mine, as well as the Technology and $\mathrm{Hu}-$ man Resources for Industry Programme (THRIP) managed by the National Research Foundation (NRF) and financed by the Department of Trade and Industry in South Africa.

\section{References}

[1] Thompson RJ, Visser AT, Miller RE, Lowe T. The development of a real-time mine road maintenance management system using haul truck and road vibration signature analysis. In: Eighth international conference on low volume roads, Nevada; 2003.

[2] Thompson RJ, Visser AT. Mine haul road maintenance management systems. J South African Inst Mining Metallurgy 2003: 303-12.

[3] Cebon D. Handbook of vehicle road interaction. Lisse: Swets \& Zeitlinger; 1999.

[4] Els PS. Die hitteprobleem op hidropneumatiese veer-en-demperstelsels. [The heat problem on hydro-pneumatic spring and damper systems]. Master's dissertation. Pretoria: University of Pretoria; 1993.

[5] Park S, Popov AA, Cole DJ. Influence of soil deformation on off-road heavy vehicle suspension vibration. J Terramech 2004;41: 41-68.

[6] Gillespie TD. Fundamentals of vehicle dynamics. Warrendale: Society of Automotive Engineers; 1992. 